\title{
A preliminary study of the mechanism of nitrate-stimulated remarkable increase of rifamycin production in Amycolatopsis mediterranei $\mathrm{U} 32$ by RNA-seq
}

\author{
Zhi Hui Shao', Shuang Xi Ren' ${ }^{1}$ Xin Qiang Liu', Jian Xu², Han Yan' ${ }^{1}$ Guo Ping Zhao ${ }^{1,3,4,5}$ and Jin Wang ${ }^{1 *}$
}

\begin{abstract}
Background: Rifamycin is an important antibiotic for the treatment of infectious disease caused by Mycobacteria tuberculosis. It was found that in Amycolatopsis mediterranei U32, an industrial producer for rifamycin SV, supplementation of nitrate into the medium remarkably stimulated the yield of rifamycin SV. However, the molecular mechanism of this nitrate-mediated stimulation remains unknown.

Results: In this study, RNA-sequencing (RNA-seq) technology was employed for investigation of the genome-wide differential gene expression in U32 cultured with or without nitrate supplementation. In the presence of nitrate, U32 maintained a high transcriptional level of genes both located in the rifamycin biosynthetic cluster and involved in the biosynthesis of rifamycin precursors, including 3-amino-5-dihydroxybenzoic acid, malonyl-CoA and (S)-methylmalonyl-CoA. However, when nitrate was omitted from the medium, the transcription of these genes declined sharply during the transition from the mid-logarithmic phase to the early stationary phase. With these understandings, one may easily propose that nitrate stimulates the rifamycin SV production through increasing both the precursors supply and the enzymes for rifamycin biosynthesis.
\end{abstract}

Conclusion: It is the first time to thoroughly illustrate the mechanism of the nitrate-mediated stimulation of rifamycin production at the transcriptional level, which may facilitate improvement of the industrial production of rifamycin SV, e.g. through optimizing the global rifamycin biosynthetic pathways on the basis of RNA-seq data.

Keywords: Amycolatopsis mediterranei, Rifamycin SV, Nitrate, RNA-seq, Transcriptome

\section{Background}

Amycolatopsis mediterranei $\mathrm{U} 32$ is an important industrial strain for production of rifamycin SV, whose derivative, rifampicin is one of the most commonly used drugs for curing mycobacterial infections [1]. It was previously found that addition of nitrate into the fermentation medium remarkably stimulated the production of rifamycin SV by $170 \%$ (abbreviated as the "nitrate stimulating

\footnotetext{
${ }^{*}$ Correspondence: jinwang@sibs.ac.cn

${ }^{1}$ CAS Key Laboratory of Synthetic Biology, Institute of Plant Physiology and Ecology, Shanghai Institutes for Biological Sciences, Chinese Academy of Sciences, Shanghai 20032, China

Full list of author information is available at the end of the article
}

effect" [2]), along with many other global physiological effects, including improving the enzymatic activities of nitrate/nitrite reductases (NR/NiR), glutamine synthetase (GS) and a few intermediate metabolic enzymes and decreasing the intracellular amount of fatty acids [2]. Besides of rifamycin SV, this "nitrate stimulating effect" is also widely applied in industrial production of many other antibiotics, e.g. lincomycin and lividomycin [3, 4]. And because of its great importance in industrial application, research into the molecular mechanism of "nitrate stimulating effect" has never stopped [2, 4-12].

Earlier interests and intensive investigation mainly focused on identification of genes involved in the effect, many of which were related to nitrogen metabolisms 
$[8-10,13,14]$. With the supplementation of nitrate, U32 mainly uses the GS/GOGAT (glutamate synthase, GOGAT) pathway for nitrogen assimilation. While under nitrogen excess, i.e. with large amount of ammonia, the alanine dehydrogenase (AlaDH, encoded by ald) instead of the commonly used glutamate dehydrogenase is adopted for ammonium assimilation [11]. It is found that nitrate increases the GS activities but decreases the activities of AlaDH. Meanwhile, the yield of rifamycin SV is positively correlated with the activities of GS but negatively correlated with the activities of AlaDH. Unlike many other actinomycetes that possess more than one GS encoding gene [15], $g \ln A$ is the only gene that encodes biologically functional GS in U32 [12]. Consistent with the measurements of enzymatic activities, nitrate remarkably activates $g \ln A$ transcription while represses ald transcription, and this transcriptional regulation is conducted by $\mathrm{Gln} R$, which is a central regulator for nitrogen metabolism in U32 $[8,10,11,16]$.

In addition, GS in U32 is regulated not at the posttranslational level, i.e. by adenylylation [12], but at the transcriptional level in response to nitrogen availability $[5,16]$. Besides $g \ln A$, nitrate also regulates the transcription many other nitrogen metabolism associated genes in U32 [8, 10, 11, 16]. However, apart from nitrogen metabolism, little is known about the nitrate-mediated global transcriptional network and the detailed mechanism of nitrate mediated stimulation of rifamycin SV production.

Transcriptome analysis, which studies all RNA transcripts under a particular physiological condition, has played an important role in detecting the global gene expression and transcriptional regulation [17]. RNAsequencing (RNA-seq) technology uses deep-sequencing technologies to directly determine the cDNA sequence and owns single-base resolution, allowing for the detection of various transcriptional features of transcript structure, operon linkages and absolute abundance $[18,19]$. In this work, RNA-seq was used to study the transcriptomic change of $A$. mediterranei $\mathrm{U} 32$ in response to nitrate supplementation. Total RNAs from two representative time points were analyzed, including the $24 \mathrm{~h}$ representing the mid-logarithmic (ML) phase and the $48 \mathrm{~h}$ representing the early stationary (ES) phase. Real-time Reverse Transcription-Polymerase Chain Reaction (RT-PCR) was further employed to quantitatively measure the expression of several important functional genes, e.g. genes responsible for both the rifamycin SV precursors supply and its biosynthesis. Based on these findings, a mechanism was finally proposed for the "nitrate stimulating effect" subject to further investigation.

\section{Results and discussion}

Influence of nitrate supplementation on both physiology and rifamycin SV production of $A$. mediterranei U32

To study the influence of extracellular nitrogen sources on rifamycin SV production, A. mediterranei U32 were cultured in nutrient-rich liquid Bennet medium with or without extracellular nitrogen sources supplemented (Figure 1). Three different nitrogen sources were tested, including $80 \mathrm{mM}$ nitrate, $60 \mathrm{mM}$ ammonium and $80 \mathrm{mM}$ mixed ammonium and nitrate. According to the growth curves, in comparison with the growth in Bennet medium, addition of ammonium slightly repressed the bacterial growth, while both nitrate and the mixed nitrogen source promoted the bacterial growth. However, only nitrate was found to remarkably stimulate the yield of rifamycin SV, which was consistent with our previous findings [2]. Obviously, the total nitrogen could not be the limiting factor for the rate of bacterial growth and rifamycin SV production, because in the conditions of ammonium and mixed nitrogen supplementation, the total nitrogen as well as its consumption rate were the same as those under the condition of nitrate addition (Figure 1e).

In addition, the glucose consumption rate and $\mathrm{pH}$ value in the medium were also measured. For the glucose consumption rate, no significant difference could be found among different culture media. The $\mathrm{pH}$ values were almost unchanged in all tested conditions except the mixed nitrogen source, in which the $\mathrm{pH}$ value approximately decreased from 7 to 5 (Figure 1c). It is still unclear whether the decrease of $\mathrm{pH}$ value has any influence on the biosynthesis of rifamycin SV.

In the presence of nitrate, the growth of U32 could be divided into two distinct developmental stages, i.e. the primary metabolism stage for vegetative growth (from 12 to $36 \mathrm{~h}$ ) and the secondary metabolism stage for production of rifamycin SV (from 36 to $72 \mathrm{~h}$ ) (Figure 1). To quantitatively measure the contribution of biomass increase and global metabolic regulation in the increase of the rifamycin SV yield, the values of specific production rate $\left(q_{P}\right)$ of rifamycin SV between Bennet and Bennet supplemented with nitrate were calculated. When fermentation process finished, the overall $q_{P}$ value $\left(\mathrm{mg}_{\text {product }} /\right.$ $\left.\left(\mathrm{mg}_{\text {dry weight }} \mathrm{h}\right)\right)$ was 0.50 for U32 grown with nitrate, while was only 0.003 when nitrate was omitted from the medium. Therefore, nitrate stimulates the production of rifamycin SV mainly through regulation of its production rate other than by increasing the bacterial biomass.

\section{RNA-seq analysis of the transcriptome of U32 grown in Bennet medium with or without nitrate supplementation}

RNA-seq was employed to reveal the nitrate-induced transcriptomic changes in U32. Samples were taken at 


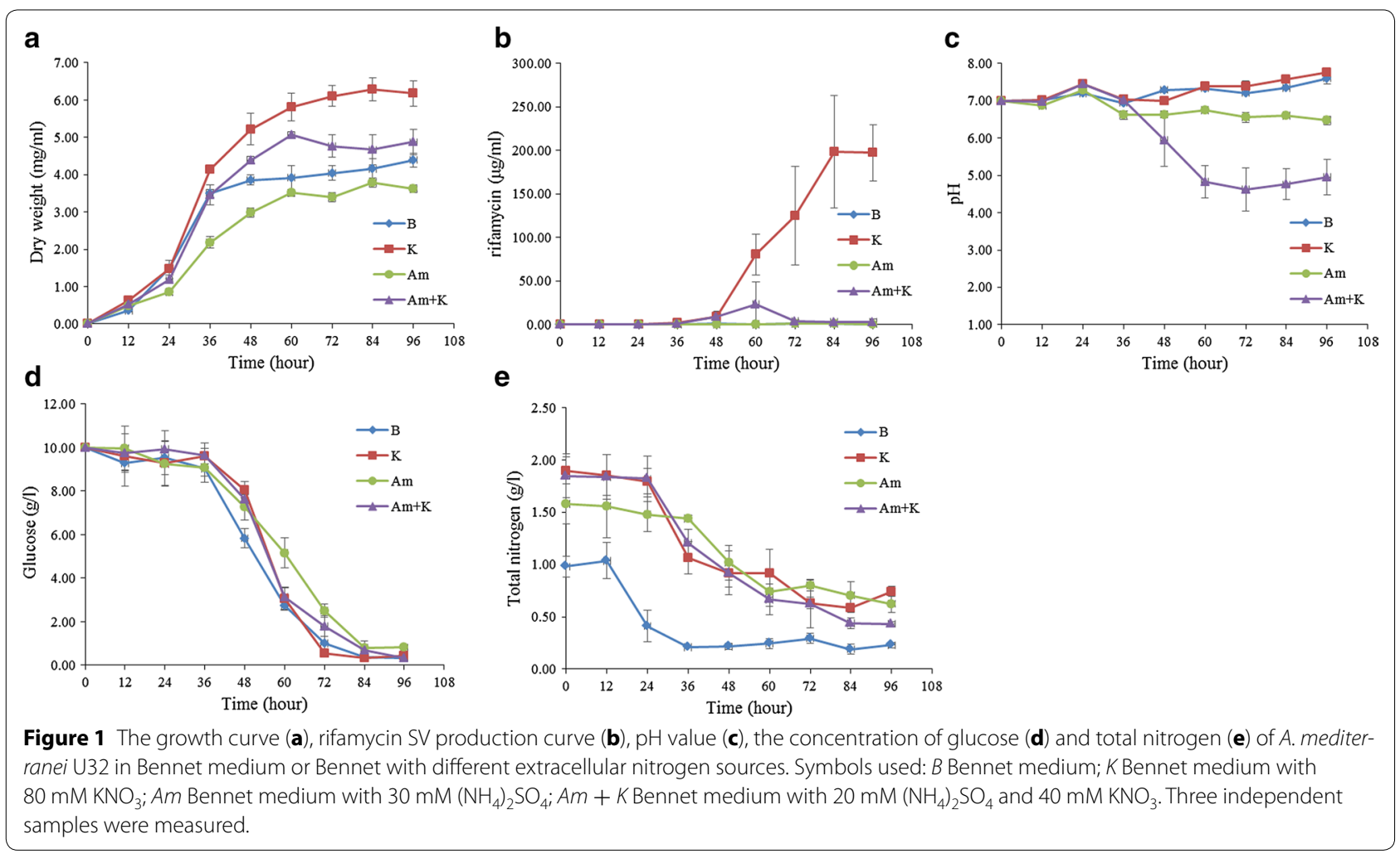

24 and $48 \mathrm{~h}$, representing the ML and ES phases, respectively. Before RNA-seq, ribosomal RNA was successfully removed with high mapping ratio obtained (Table 1). Annotated genes include 9228 CDSs, 12 rDNA genes and 52 tRNA-encoding genes [9] and nearly $70 \%$ of them expressing with RPKM $>5$ at two tested time points (i.e. ML and ES phases) either with or without nitrate supplementation (Table 1). On the basis of RNA-seq data, reannotation of U32 genome information was performed, with the newly identification of the Valyl-tRNA synthetase encoding gene (AMED_6793.1) that was missed from previous annotations [9]. In addition, with the use of Rockhopper $[20,21]$ by setting reads more than 10 and length between 50 and 500 nucleotides, we identified a large number of noncoding RNAs (ncRNAs), including 600 intergenic transcripts and 582 antisense transcripts, which comprised of about $11.29 \%$ of total transcripts. It is well-known that ncRNAs are involved in the regulation of various processes such as transcriptional interference, translational control and regulation of mRNA half-life [22, 23]. In U32, the specific physiological functions of these ncRNAs, especially in regulation of secondary metabolisms, are unclear, and it is an interesting direction to delineate their physiological roles in future studies.

In response to nitrate supplementation, both the number and fold change of differentially expressed genes in ES phase were apparently higher than those in ML phase (Figure 2). For example, using statistical criteria of FDR $<0.05$ (FDR, false discovery rate control) from Fisher test and FC $>2$ (FC, fold change), there were totally 1,880 genes transcriptionally regulated in ES phase, while only

Table 1 Transcriptomic profiles of $A$. mediterranei U32 in different culture conditions

\begin{tabular}{lllllll}
\hline Sample $^{\mathbf{a}}$ & Raw reads & Mapped reads & Mapping ratio (\%) & Genes (RPKM >5) & Expression ratio (\%) & Differential genes $^{\mathbf{b}}$ \\
\hline 24B & $18,335,500$ & $17,101,379$ & 93.27 & 6479 & 69.72 & 219 up/334 down \\
24K & $17,343,634$ & $16,514,363$ & 95.22 & 6498 & 69.92 & 1,033 up/847 down \\
$48 \mathrm{~B}$ & $18,740,965$ & $17,564,533$ & 93.72 & 6324 & 68.05 & 69.76 \\
$48 \mathrm{~K}$ & $27,441,452$ & $24,162,480$ & 88.05 & 6483 & \\
\hline
\end{tabular}

a 24B, $24 \mathrm{~h}$ in Bennet medium; 24K, $24 \mathrm{~h}$ in Bennet medium with $80 \mathrm{mM}$ nitrate; 48B, $48 \mathrm{~h}$ in Bennet medium; 48K, $48 \mathrm{~h}$ in Bennet medium with $80 \mathrm{mM}$ nitrate. Mapping ratio: mapped reads/raw reads. Expression ratio: genes (RPKM $\geq 5) / 9,293$ genes.

b Genes expressed under nitrate conditions were compared to those in Bennet medium. 

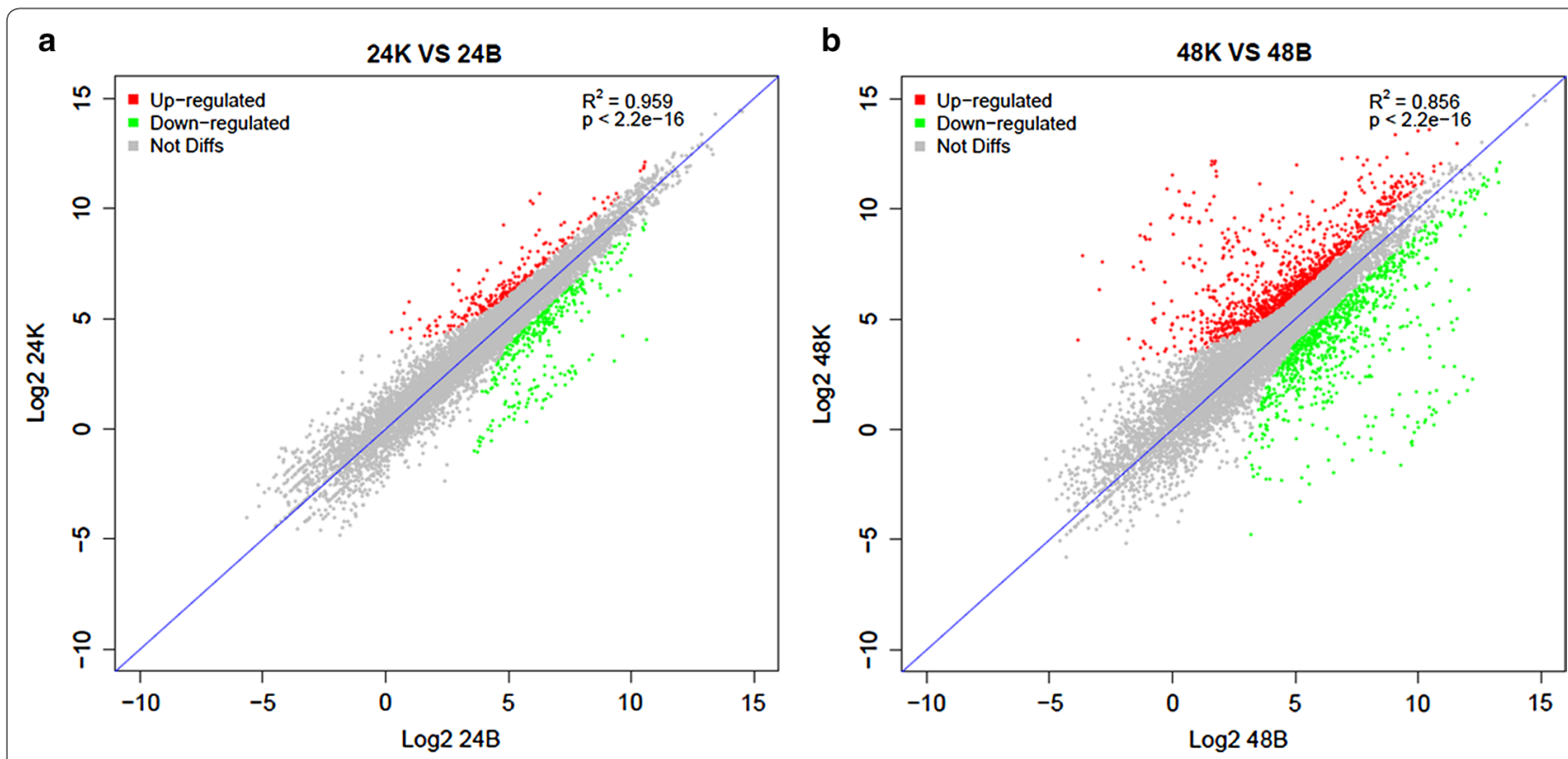

Figure 2 Correlation of RNA expression levels between two nitrogen conditions in two phases of U32. RNA expression levels per gene were presented as RPKM. a Correlation of RNA expression levels in $24 \mathrm{~h}$ (ML phase) for U32 in Bennet medium with ( $K$ ) or without $\mathrm{KNO}_{3}(B)$; $\mathbf{b}$ correlation of RNA expression levels in $48 \mathrm{~h}$ (ES phase). Test was considered to be significant when $p$ value was $<0.05$.

553 genes were found in ML phase (Table 1). Actually, actinomycetes such as $A$. mediterranei usually transit from the primary metabolism into the secondary metabolism in ES phase and this transcriptionally regulatory pattern at the two growth phases was likely to be consistent with the physiological changes.

According to clusters of orthologous group (COG), functional genes in U32 were divided into 24 categories [9]. Using the Wilcoxon test, 8 categories obviously changed at the transcriptional level in ML phase and 13 categories changed in ES phase in response to nitrate supplementation (Table 2). Consistent with the bacterial physiological growth, the transcriptionally changed categories were mainly related to the primary metabolisms in ML phase, while differential expression of genes related to secondary metabolisms such as the biosynthesis of rifamycin SV were observed in ES phase. Therefore, comparative analyses of the global transcriptional profiling in ES phase, i.e. with versus without nitrate supplementation, were performed in this work to reveal the molecular mechanisms of the nitrate-mediated stimulation of rifamycin SV production.

\section{Nitrate supplementation stimulates the precursor supplies in rifamycin SV biosynthesis}

Genes responsible for the biosynthesis of key precursors for rifamycin SV, including 3-amino-5-dihydroxybenzoic acid (AHBA), malonyl-CoA and (S)-methylmalonyl-CoA, were significantly regulated in ES phase responding to nitrate supplementation (Figure 3 ).

AHBA is synthesized step-by-step via enzymes encoded by genes of rifGHIJKLMN and AMED_0651$A M E D \_0652$, employing substrates of UDP-glucose and glutamine. Upon nitrate addition, the transcription of these genes was up-regulated to $24.6,25.5,25.5,55.9$, 466.3, 237.0, 24.5, 10.4, 1,354.0 and 977.7 folds, respectively (Additional file 1: Table S1). Compared to conditions without nitrate supplementation, nitrate increased the expression of gall gene, which encodes the UDPglucose pyrophosphorylase catalyzing the biosynthesis of UDP-glucose, by 6.8 folds (Figure 3). Meanwhile, the synthesis of glutamine, which is a key substrate for AHBA biosynthesis and provides the only nitrogen atom in rifamycin SV, was also remarkably enhanced by addition of nitrate. Genes involved in this process were activated at the transcriptional level, including the nas $A C K B D E$ operon encoding proteins responsible for nitrate assimilation and $g \ln A$ encoding the only GS with biological functions $[8,9,24]$ (Additional file 1: Table S1; Figure 3). Nitrate activated the expression of nas $A C K B D E$ operon and $g \ln A$, i.e. by $1,399.4,2,910.6,779.0,1,189.1,465.5$ and 463.3 folds for nas $A C K B D E$ genes and by 13.0 folds for $\operatorname{gln} A$ (Figure 3 ), which was in well consistence with previous findings $[8,16]$.

In addition, totally two malonyl-CoA molecules (C2 extender unit) and eight (S)-methylmalonyl-CoA (C3 
Table 2 Wilcoxon test-assisted statistical analysis of the expression of grouped genes according COG

\begin{tabular}{|c|c|c|c|c|}
\hline \multirow[t]{2}{*}{ COG } & \multirow[t]{2}{*}{ Description } & \multirow[t]{2}{*}{ The number of genes } & \multicolumn{2}{|l|}{$P$ value } \\
\hline & & & $24 \mathrm{~h}$ & $48 \mathrm{~h}$ \\
\hline A & RNA processing and modification & 2 & 0.5000 & 0.5000 \\
\hline B & Chromatin structure and dynamics & 2 & 1.0000 & 1.0000 \\
\hline C & Energy production and conversion & 711 & 0.7816 & 0.2295 \\
\hline D & Cell cycle control, cell division, chromosome partitioning & 55 & 0.9407 & $0.0057^{*}$ \\
\hline $\mathrm{E}$ & Amino acid transport and metabolism & 1,072 & $0.0003^{*}$ & $<0.0001^{*}$ \\
\hline $\mathrm{F}$ & Nucleotide transport and metabolism & 232 & $<0.0001^{*}$ & $<0.0001^{*}$ \\
\hline G & Carbohydrate transport and metabolism & 889 & $0.0012^{*}$ & 0.6980 \\
\hline H & Coenzyme transport and metabolism & 475 & 0.9892 & 0.1238 \\
\hline । & Lipid transport and metabolism & 637 & 0.9888 & $<0.0001^{*}$ \\
\hline J & Translation, ribosomal structure and biogenesis & 268 & $<0.0001^{*}$ & $<0.0001^{*}$ \\
\hline K & Transcription & 1,155 & $<0.0001^{*}$ & $<0.0001^{*}$ \\
\hline L & Replication, recombination and repair & 265 & $<0.0001^{*}$ & $0.0085^{*}$ \\
\hline M & Cell wall\membranelenvelope biogenesis & 515 & 0.1580 & $0.0056^{*}$ \\
\hline N & Cell motility & 43 & 0.0589 & 0.2363 \\
\hline O & Posttranslational modification, protein turnover, chaperones & 285 & 0.5207 & 0.1124 \\
\hline P & Inorganic ion transport and metabolism & 544 & $0.0040^{*}$ & $<0.0001^{*}$ \\
\hline Q & Secondary metabolites biosynthesis, transport and catabolism & 636 & 0.2382 & $<0.0001^{*}$ \\
\hline $\mathrm{R}$ & General function prediction only & 1,646 & $0.0028^{*}$ & $0.0017^{*}$ \\
\hline S & Function unknown & 571 & 0.6361 & $0.0007^{*}$ \\
\hline $\mathrm{T}$ & Signal transduction mechanisms & 540 & 0.3348 & $0.0014^{*}$ \\
\hline$U$ & Intracellular trafficking, secretion, and vesicular transport & 58 & 0.7051 & 0.5160 \\
\hline V & Defense mechanisms & 201 & 0.0637 & 0.6362 \\
\hline W & Extracellular structures & 1 & 1.0000 & 1.0000 \\
\hline Z & Cytoskeleton & 3 & 0.5000 & 1.0000 \\
\hline
\end{tabular}

* Difference is regarded as significant when P value is smaller than 0.01 .

extender unit) are needed for polyketide chain elongation during the biosynthesis of rifamycin SV [2]. In U32, malonyl-CoA is reversibly converted from acetyl-CoA by the acetyl-CoA carboxylase (Acc). Upon supplementation of nitrate, no obvious difference was found in the expression of acc genes. However, the expression of the fatty acid synthase (FAS), which is a hexamer complex encoded by the $f a b$ operon, was down regulated after nitrate supplementation. In particular, the FabG-encoding genes, AMED_5426 and AMED_5430, both of which expressed more than tenfold higher than other $f a b$ genes in ES phase (Additional file 1: Table S1), were transcriptionally repressed by threefold after addition of nitrate. As the fatty acids and rifamycin SV share the same common precursor of malonyl-CoA, one may easily propose that nitrate addition shifts the malonyl-CoA flux from the fatty acid biosynthesis to rifamycin SV biosynthesis. Meanwhile, these data were consistent with our previous findings that nitrate repressed the intracellular lipid biosynthesis in $A$. mediterranei when observed with electron microscopy [2].

There are totally three pathways for the biosynthesis of C3 extender unit of $(S)$-methylmalonyl-CoA, including the propionyl-CoA carboxylase (Pcc), methylmalonylCoA carboxyltransferase (Mct) and methylmalonylCoA mutase (Mcm)-methylmalonyl-CoA epimerase (Mce) pathways. Based on previous enzymatic studies [25], the Mcm-Mce pathway is the main pathway for C3 extender unit biosynthesis in U32. Consistently, no transcriptional difference was observed for Pcc and Mct encoding genes, both of which were annotated as $p c c$ or acc genes, under two culture conditions.

For the Mcm-Mce pathway, succinyl-CoA is firstly reversibly catalysed into $(R)$-methylmalonyl-CoA by Mcm [26], which is then converted into $(S)$-methylmalonyl-CoA by Mce. There are totally four $\mathrm{mcm}$ genes annotated in U32, including AMED_0913, AMED_0914, $A M E D \_2498$ and $A M E D \_7761$, among which $A M E D \_2498$ was apparently transcriptionally repressed by as much as 25 folds after the addition of nitrate while the transcription of others was almost unchanged in ES phase (Additional file 1: Table S1). The mutB2, encoding the large subunit of $\mathrm{Mcm}$, is the key gene for accumulation of intracellular methylmalonyl-CoA $[27,28]$ and mutation of mutB2 in A. mediterranei HP-130, an 


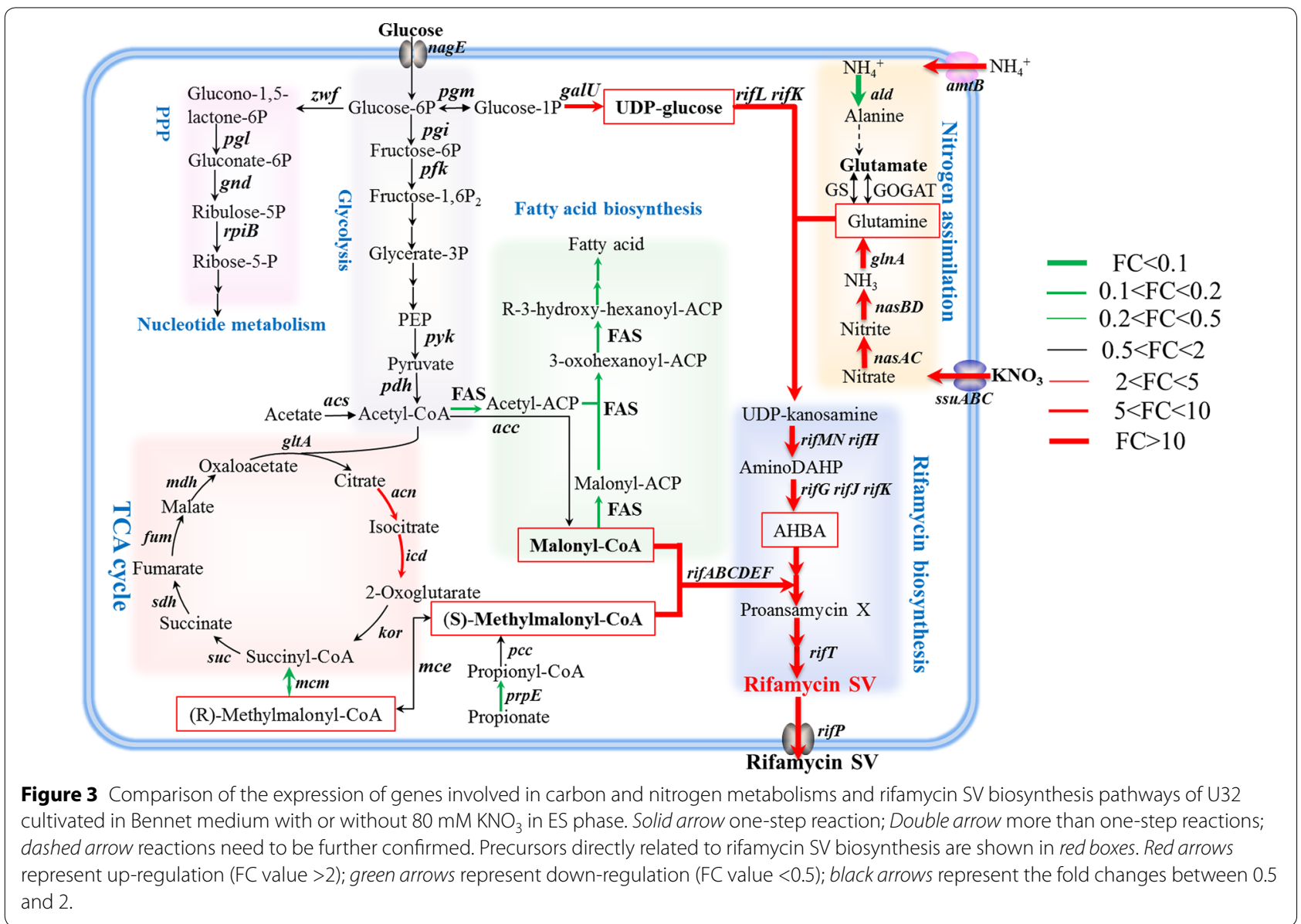

overproducer of rifamycin $\mathrm{B}$, significantly increased the yield [29]. Notably, the mutB2 homologue, AMED_7761 kept an extremely low transcriptional level in U32, which may account for the high yield of rifamycin SV. Moreover, the transcription of mce was up-regulated 1.4 folds upon addition of nitrate, which would therefore lead to enhanced synthesis of the C3 extender unit, (S)-methylmalonyl-CoA.

\section{Nitrate supplementation activates the transcription of the rifamycin SV biosynthetic genes}

Besides of the AHBA biosynthetic genes, i.e. rifG through rifN $[30,31]$, the transcripts of other important genes in rif cluster in ES phase raised several folds (ranging from 7.6 to 2,960.3 folds) higher in nitrate conditions than those in the absence of nitrate. As the products of these genes are responsible for the biosynthesis of rifamycin $\mathrm{SV}$, e.g. rifABCDE encode a type I polyketide synthase (PKS) module; several genes encode post-PKS modification enzymes and rifP encodes the rifamycin exporter $[32,33]$ (Figures 4, 5), nitrate supplementation apparently increases the rifamycin SV biosynthetic enzymes.
To validate the RNA-seq data, real-time RT-PCR was used to quantitatively measure the expression of genes involved in both precursors supply and rifamycin SV biosynthetic enzymes. Selected genes include six genes in carbon metabolism such as korA, sucC, $m c m, \operatorname{acn} A$, fabG and galU, four genes involved in nitrogen metabolism such as nas $A, g \ln A, a m t B$ and ald, and four genes involved in rifamycin SV biosynthesis such as rifA, rifK, rif16 and $\operatorname{lux}$. Four time points of 12, 24, 36 and $48 \mathrm{~h}$ were selected for the real-time RT-PCR analysis with transcriptional results in good accordance with the RNAseq data (Figure 5).

Therefore, a molecular mechanism has been proposed for the "nitrate stimulating effect". Nitrate obviously activates the transcription of genes responsible for both the precursors supply and the rifamycin SV biosynthesis, which consequently enhances the yield of rifamycin SV. Similarly, in A. mediterranei HP-130, an industrial strain for high yield of rifamycin B, rif cluster kept continuously higher expression than its reference strain S699 [29].

Besides the rif cluster, the transcription of 19 other gene clusters [9] for secondary metabolisms was also 


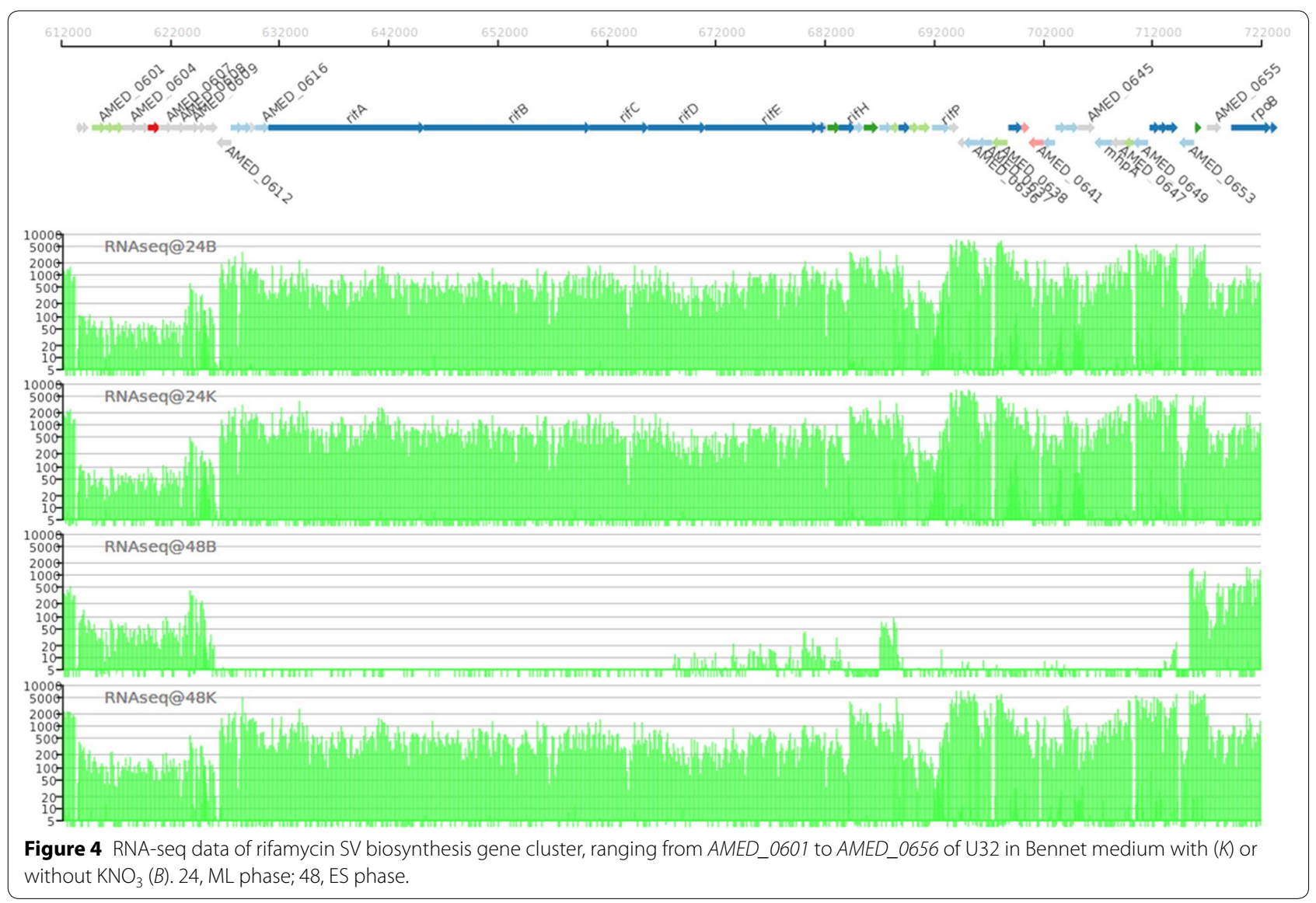

investigated (Additional file 2: Table S2). Among them, 11 clusters were found to transcribe at an extremely low level $($ RPKM $<5)$. Three clusters were transcriptionally repressed by nitrate addition, including nrps_pks1, nrps 8 and nrps9, while only nrps6 and nrps10 were transcriptionally activated. Compared to other actinomycetes, e.g., Streptomyces coelicolor [34], there are extremely few highly transcribed clusters in U32 under nitrate supplemented condition, which may therefore favor the production of rifamycin SV. In addition, because genes involved in the Glycolysis pathway and TCA cycle, including pyc (3.5 folds), acn (2.6 folds), icd (2.9 folds) and $a c e B$ (2.6 folds), were transcriptionally activated by addition of nitrate, the energy supply could also be enhanced under this condition, probably facilitating the biosynthesis of rifamycin SV.

\section{Future perspectives in studying the mechanism} of nitrate-mediated global transcriptional regulation

Nitrate has been considered as a poor nitrogen source for bacteria such as Escherichia coli, while for plants and algae [35], it is well known as a preferred nitrogen source [36, 37]. For A. mediterranei U32, nitrate activates the transcription of a series of genes involved in nitrogen assimilation, e.g. $g \ln A$, nas operon and $a m t B$, while represses the expression of ald, even in rich Bennet medium. Therefore, considering the transcriptional regulation of these nitrogen metabolism-related genes, one may conclude that Bennet medium with nitrate supplementation mimics a poor nitrogen source for U32 [11]. However, compared to ammonium, nitrate obviously stimulates the growth of U32 in both rich Bennet medium and minimal medium [7]. Therefore, "how would the poor-nitrogen nitrate promote the growth of U32" would be an interesting question subject to further investigation.

GlnR has been characterized as a central regulator for nitrogen metabolisms in several actinomycetes, with $A$. mediterranei U32 included [10, 11, 16, 38, 39]. The transcriptional regulation of nitrogen metabolisms-related genes, including $g \ln A$, nas operon and ald, which are closely responsible for the accumulation of glutamine, is under stringent regulation by $G \ln R[10,11,16]$, responding to extracellular nitrogen availabilities. However, little is known about the transcriptional regulation of other genes that provide precursors for rifamycin SV biosynthesis, e.g. fabG, $\mathrm{mcm}$ and gall . As these genes are involved in important pathways of carbon metabolism 


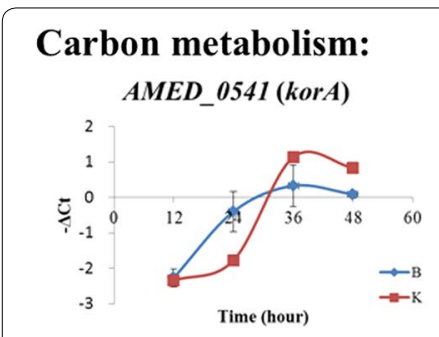

AMED $4402(a r a B)$

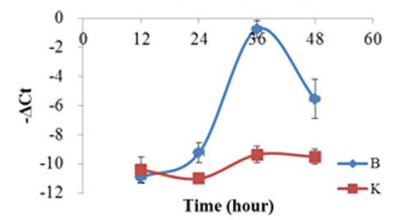

Nitrogen metabolism:
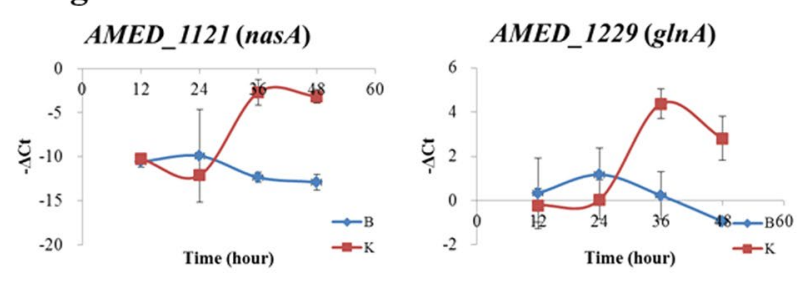

AMED_0802(sucC)

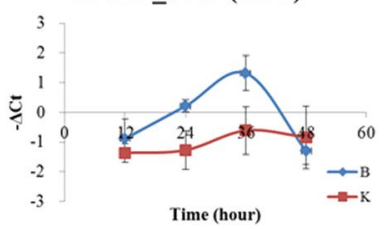

AMED 4955 (acnA)

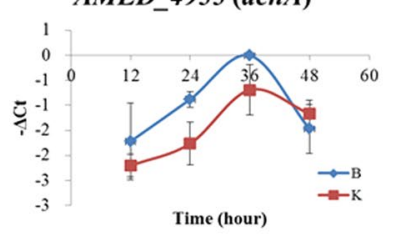

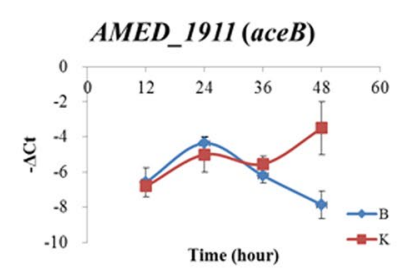

AMED_5426(fabD)

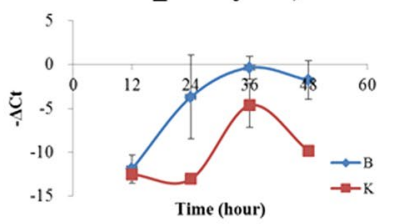

AMED_2498(mcm)

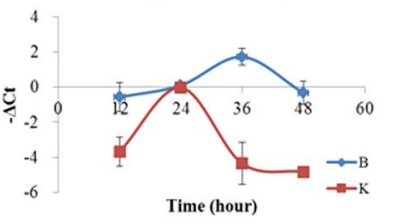

AMED_8311 (gall)

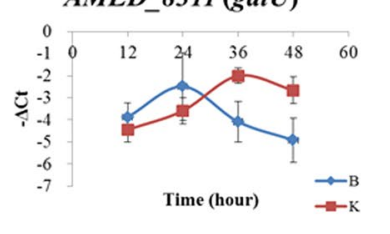

\section{Rifamycin biosynthesis:}
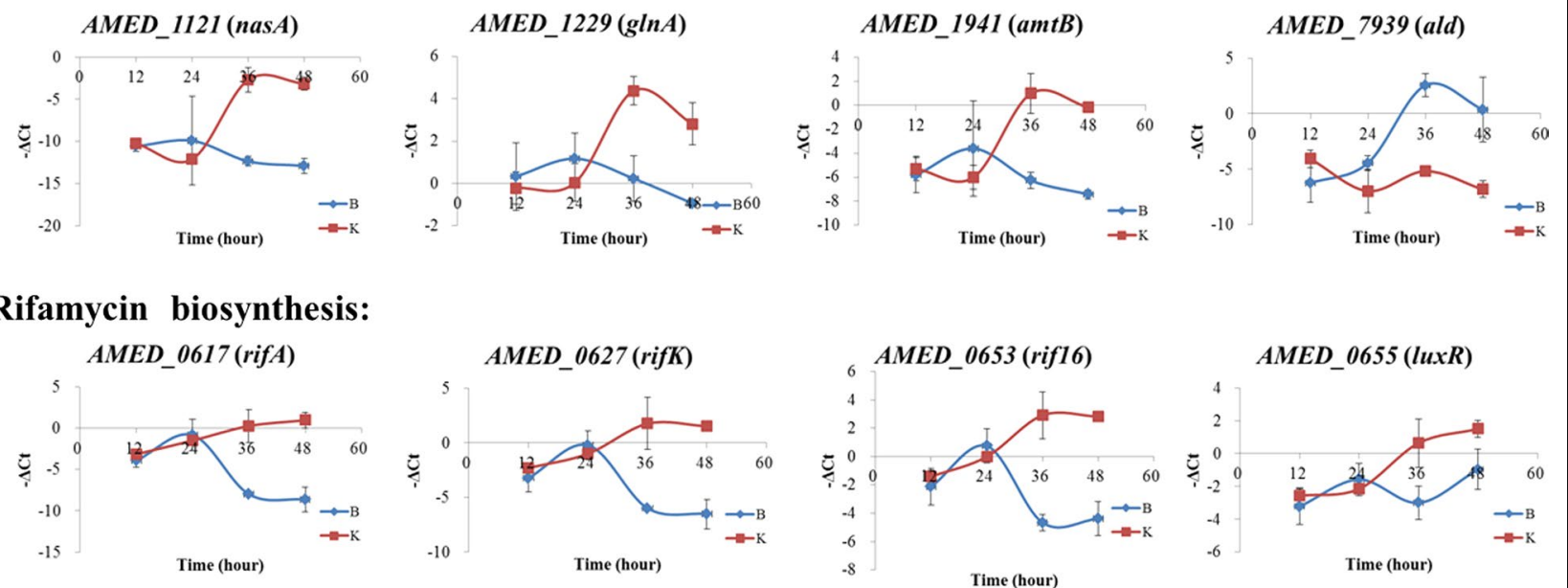

Figure 5 Time-course results of real-time PCR of key genes involved in rifamycin SV biosynthesis for U32 cultivated in Bennet medium with ( $K$ ) or without $\mathrm{KNO}_{3}(B)$. The transcription of $r p o B$ is used as the internal control and error bars represent the standard deviation of three biological replicates.

but are transcriptionally regulated by extracellular nitrogen sources, research into their transcriptional regulation may provide an important clue for study of the coordination of carbon and nitrogen metabolisms in U32.

In addition, the regulation of the transcription of rif cluster seems more complex. In ML phase, the transcription of rif genes remains at a high level with or without the supplementation of extracellular nitrate. However, when cells enter the ES phase, the transcription of rif genes only slightly increased under the condition of nitrate, but decreased dramatically in the absence of nitrate (Figures 4, 5). Therefore, nitrate either prolongs the transcriptional period of rif cluster till ES phase or merely helps maintain the stability of the riftranscripts. And further delineation of the detailed mechanisms, including identification of regulators/proteins involved, would be an interesting project.
Moreover, nitrate-mediated global regulation must be multi-level, which may include transcriptional, translational and post-translational levels. And for those important genes that are not regulated at the transcriptional level, there may be post-transcriptional regulations, which will be a focus of research in our future work.

\section{Conclusions}

In this work, we in-depth studied the "nitrate-stimulating effect" in U32 at the transcriptional level, based on which a molecular mechanism was successfully proposed. We noticed that most genes involving in both the precursor production and rifamycin SV biosynthesis were transcriptionally activated by nitrate addition, well explaining the nitrate-mediated activation of rifamycin SV production. 


\section{Methods}

\section{Strains and culture conditions}

Amycolatopsis mediterranei U32 (CGMCC 4.5720) was grown aerobically at $30^{\circ} \mathrm{C}$ in rich Bennet medium (glucose $1.0 \%$, tryptone $0.2 \%$, yeast extract $0.1 \%$, beef extract $0.1 \%$, glycerol $1.0 \%, w / v, p H 7.0)$ [2, 40]. After 48 h's culturing, $5 \%$ of U32 cells was inoculated into fresh liquid Bennet medium or Bennet with extracellular nitrogen sources supplemented, i.e. $80 \mathrm{mM}$ potassium nitrate, $30 \mathrm{mM}$ ammonium sulphate [5] or $80 \mathrm{mM}$ mixed nitrate and ammonium. For mixed nitrogen source, $40 \mathrm{mM}$ potassium nitrate and $20 \mathrm{mM}$ ammonium sulphate were used to make up $80 \mathrm{mM}$ nitrogen. Cells were harvested at different time points $(12,24,36$ and $48 \mathrm{~h})$ by centrifugation at $12,000 \mathrm{rpm}$ for $5 \mathrm{~min}$ at $4^{\circ} \mathrm{C}$ and were then quickly frozen in liquid nitrogen for total RNA isolation.

\section{Measurement of growth rate, rifamycin SV production, $\mathrm{pH}$ value, glucose consumption and total nitrogen in medium} The growth curves were determined through measuring the bacterial dry weight. The $\mathrm{pH}$ value in the medium was measured by a $\mathrm{pH}$ meter (Mettler Toledo). The glucose concentration was determined with an enzymatic test kit (Rsbio, Shanghai, China), following the manufacturer's instructions. The total nitrogen concentration (as nitrate-N) was measured with a colorimetric way, the same as that described by Ferree et al. [41].

Rifamycin SV was measured as previously described $[9,13]$. Strains were cultured for 4 days in Bennet liquid medium at $30^{\circ} \mathrm{C}$. Samples of the culture broths were taken with every $24 \mathrm{~h}$ internals, adjusted to $\mathrm{pH} 2-3$ with $1 \mathrm{M} \mathrm{HCl}$ and extracted once with equal volumes of ethyl acetate. Ethyl acetate solutions were filtered $(0.22 \mu \mathrm{m})$ and then directly analyzed by HPLC (Agilent HPLC 1260 Infinity) with detection wavelength at 280 and $447 \mathrm{~nm}$, employing the 95\% pure rifamycin SV (Sigma-Aldrich) as a reference standard. The column was an Agilent poroshell 120 EC-C18 column $\left(4.6 \times 50 \mathrm{~mm}, 2.7 \mathrm{~mm}, 30^{\circ} \mathrm{C}\right)$ and the mobile phase was $70 \%$ methanol in water supplemented with $0.1 \%$ formic acid with a flow rate of $1.0 \mathrm{ml} / \mathrm{min}$. All data were presented as mean \pm standard deviations for the number of experiments indicated in each case.

The values of specific production rate $\left(q_{P}: \mathrm{mg}_{\text {product }}\right)$ $\left.\left(\mathrm{mg}_{\text {dry weight }} \cdot \mathrm{h}\right)\right)$ of rifamycin SV between two growth conditions were calculated according to the following Eqs. (1) and (2) [ $\rho_{X}$ : cell concentration $(\mathrm{mg} / \mathrm{ml}) ; \rho_{P}$ : product concentration $(\mathrm{mg} / \mathrm{ml})$; t: culture time $(\mathrm{h})]$

$$
\begin{aligned}
& q_{P}=\frac{1}{\rho_{X}} \cdot \frac{d \rho_{P}}{d t} \\
& q_{P}=\frac{\rho_{P j}}{\sum_{i=1}^{j} \frac{\rho_{x i}+\rho_{x(i-1)}}{2} \times\left(t_{i}-t_{i-1}\right)} j=\frac{t}{12}
\end{aligned}
$$

\section{Sample preparation for RNA-seq and sequencing procedures}

Bacteria were lysed by constantly grinding in the presence of liquid nitrogen and total RNA was extracted using TRIzol reagent (Invitrogen). RNA was fully treated with RNase-free DNase I (Takara) to prevent contamination of trace genomic DNA [8, 42]. The RNA quality was evaluated by the BioAnalyzer 2100 system (Agilent) and ribosomal RNAs were removed with the RiboZero rRNA removal kit (Epicenter) for gram-positive organisms prior to sequencing analysis. After deprivation of the rRNA, RNA was fragmented and used as a template for a randomly primed PCR.

Strand-specific cDNA libraries were prepared by standard techniques for subsequent Illumina sequencing using the mRNA-seq Sample Prep kit (Illumina). The concentration of the cDNA library was detected by Qubit ${ }^{\circ}$ 2.0 Fluorometer and verified for appropriate fragment size (200-300 bp) on a BioAnalyzer 2100 system (Agilent). Samples were amplified onto flowcells using an Illumina cBot and sequenced on an Illumina HiSeq 2500 for 51 cycles according to manufacturer protocols. Raw sequencing data was processed using the data collection software provided by Illumina [43].

\section{Data analysis for RNA-seq}

Sequencing raw reads were preprocessed by filtering out rRNA reads, sequencing adapters, short-fragment reads and other low-quality reads. The rest reads were used to map to reference genome of A. mediterranei U32 at the NCBI website by Bowtie2 software (version: 2.0.5), based on the local alignment algorithm [43]. Reads aligning to multiple locations are kept (to a maximum of 20 potential positions) to assist constructing gene models for genes with repetitive or low complexity features. When aligning reads, 1 mismatch to the reference was allowed. Alignments reported from Bowtie 2 software were further processed by the BEDTools software [44] to determine the quantity of the expression of transcripts and their differential expression between conditions. Expression values were presented as Reads Per Kilobase of Gene Per Million Mapped Reads (RPKM). Small non-coding RNAs were identified by Rockhopper software [20, 21]. Data were visualized using the Integrated Genomics Viewer. Differential expression of all of transcripts was quantified through DEGseq software (version: 2.16.1) [45], and then the values of fold change were represented. In general, it was regarded as no distinct difference between two transcripts when their value of fold change fell in between 0.5 and 2 . At the same time, P-values were determined and significance was accurately assessed by conducting correction for multiple testing such as Fisher test and Wilcoxon test. RNA-seq data have been submitted to NCBI (accession number: GSE63313). 


\section{Quantitative real-time PCR}

Reverse transcription was performed with a random hexamer primer using $2 \mu \mathrm{g}$ total RNA in a volume of $20 \mu \mathrm{l}$ employing SuperScript III reverse transcriptase (Invitrogen). PCR was performed employing $20 \mathrm{ng}$ reaction mixtures as the template, using the $r p o B$ gene as the internal control. A negative control was performed by following the same procedures except that the addition of reverse transcriptase was omitted. Totally, 14 genes, including 6 genes related to carbon metabolisms, 4 genes related to nitrogen metabolism and 4 genes involved in rifamycin biosynthesis according to RNA-seq data, were selected for quantitative real-time PCR (qPCR) validation experiments, using a SYBR Premix Ex Taq GC kit (Takara) in a Step-one Plus real-time PCR system (Applied Biosystems).

\section{Additional files}

Additional file 1: Table S1. Transcriptional profiles of genes involved in carbon metabolism, nitrogen metabolism and rifamycin SV biosynthesis in A. mediterranei U32

Additional file 2: Table S2. Transcriptional profiles of genes for secondary metabolisms except for the rifamycin SV biosynthesis in A. mediterranei U32.

\begin{abstract}
Abbreviations
RNA-seq: RNA-sequencing; NR/NiR: nitrate/nitrite reductases; GS: glutamine synthetase; GOGAT: glutamate synthase; AlaDH: alanine dehydrogenase; RTPCR: Reverse Transcription-Polymerase Chain Reaction; ML: mid-logarithmic; ES: early stationary; AHBA: 3-amino-5-dihydroxybenzoic acid; Acc: acetyl-CoA carboxylase; FAS: fatty acid synthase; Pcc: propionyl-CoA carboxylase; Mct: methylmalonyl-CoA carboxyltransferase; $\mathrm{Mcm}$ : methylmalonyl-CoA mutase; Mce: methylmalonyl-CoA epimerase; Sdh: succinate dehydrogenase; Suc: succinyl-COA synthetase; PKS: polyketide synthase; PEP: phosphoenolpyruvic acid; aminoDAHP: 3,4-dideoxy-4-amino-d-arabino-heptulosonic acid 7-phosphate
\end{abstract}

\section{Authors' contributions}

ZS performed all experiments and drafted the manuscript. ZS and SR analyzed the sequences. $X \mathrm{~L}$ and $\mathrm{HY}$ assisted part of experiments. JX helped analysis of experiment data. GZ and JW supervised the project and revised the manuscript. All authors read and approved the final manuscript.

\section{Author details \\ ${ }^{1}$ CAS Key Laboratory of Synthetic Biology, Institute of Plant Physiology and Ecology, Shanghai Institutes for Biological Sciences, Chinese Academy of Sciences, Shanghai 20032, China. ${ }^{2}$ State Key Laboratory of Bioreactor Engineering, New World Institute of Biotechnology, East China University of Science and Technology, Shanghai 200237, China. ${ }^{3}$ State Key Lab of Genetic Engineering and Center for Synthetic Biology, Department of Microbiology and Microbial Engineering, School of Life Sciences, Fudan University, Shang- hai 200032, China. ${ }^{4}$ Shanghai-MOST Key Laboratory of Disease and Health Genomics, Chinese National Human Genome Center at Shanghai, Shang- hai 201203, China. ${ }^{5}$ Department of Microbiology and Li KaShing Institute of Health Sciences, The Chinese University of Hong Kong, Prince of Wales Hospital, Shatin, New Territories, Hong Kong Sar, China.}

\section{Acknowledgements}

This work was supported by the National Basic Research Program of China [2012CB721102] and National Natural Science Foundation of China [31200021, 31121001 and 31300031]. We acknowledge Shanghai Biotechnology Corporation for their assistance in RNA-seq work.

\section{Compliance with ethical guidelines}

\section{Competing interests}

The authors declare that they have no competing interests.

Received: 26 January 2015 Accepted: 11 May 2015

Published online: 04 June 2015

\section{References}

1. Lal R, Lal S (1994) Recent trends in rifamycin research. Bioessays 16:211-216

2. Jiao RS, Chen YM, Wu MG, Gu WL (1979) Studies on the metabolic regulation of biosynthesis of rifamycin by Norcadia (Amycolatopsis) mediterranei I. The stimulative effect of nitrate on biosynthesis of rifamycin SV by Nocardia mediterranei. Acta Phytophysiol Sin 5:395-402

3. Zhou XY, Huang WY (1995) Study of lividomycin produced by lividomycin producer M814. J Zhejiang Univ Technol 23:67-72

4. Jin Z, Jiao RS (1997) Stimulative effects of nitrate and magnesium salts on biosynthesis of lincomycin by Streptomyces lincolnensis. Chin Biochem J 13:709-715

5. Yu H, Yao Y, Liu Y, Jiao R, Jiang W, Zhao GP (2007) A complex role of Amycolatopsis mediterranei GlnR in nitrogen metabolism and related antibiotics production. Arch Microbiol 188:89-96

6. Gu WL, Lu XY, Geng YQ, Liu CJ, Jin ZK, Jiao RS (1983) The regulatory effect of nitrate on the relationship between the production of rifamycin SV and the biosynthesis of cellular lipid components. Acta Microbiol Sin 23:313-318

7. Chen YM, Gu WL, Wang W, Jiao RS (1980) Studies on the metabolic regulation of biosynthesis of rifamycin by Norcadia (Amycolatopsis) mediterranei II. The regulatory effect of nitrate on the metabolic pathway of Norcadia (Amycolatopsis) mediterranei. Acta Phytophysiol Sin 6:291-298

8. Shao Z, Gao J, Ding X, Wang J, Chiao J, Zhao G (2010) Identification and functional analysis of a nitrate assimilation operon nasACKBDEF from Amycolatopsis mediterranei U32. Arch Microbiol 193:463-477

9. Zhao W, Zhong Y, Yuan H, Wang J, Zheng H, Wang Y et al (2010) Complete genome sequence of the rifamycin SV-producing Amycolatopsis mediterranei $\mathrm{U} 32$ revealed its genetic characteristics in phylogeny and metabolism. Cell Res 20:1096-1108

10. Wang Y, Wang JZ, Shao ZH, Yuan H, Lu YH, Jiang WH et al (2013) Three of four $\mathrm{GlnR}$ binding sites are essential for GlnR-mediated activation of transcription of the Amycolatopsis mediterranei nas operon. J Bacteriol 195:2595-2602

11. Wang Y, Li C, Duan N, Li B, Ding XM, Yao YF et al (2014) GlnR negatively regulates the transcription of the alanine dehydrogenase encoding gene ald in Amycolatopsis mediterranei U32 under nitrogen limited conditions via specific binding to its major transcription initiation site. PLoS One 9:e104811

12. Peng WT, Wang J, Wu T, Huang JQ, Chiao JS, Zhao GP (2006) Bacterial type I glutamine synthetase of the rifamycin SV producing actinomycete, Amycolatopsis mediterranei U32, is the only enzyme responsible for glutamine synthesis under physiological conditions. Acta Biochim Biophys Sin (Shanghai) 38:821-830

13. Yuan H, Zhao W, Zhong Y, Wang J, Qin Z, Ding X et al (2011) Two genes, rif15 and rif16, of the rifamycin biosynthetic gene cluster in Amycolatopsis mediterranei likely encode a transketolase and a P450 monooxygenase, respectively, both essential for the conversion of rifamycin SV into B. Acta Biochim Biophys Sin (Shanghai) 43:948-956

14. Ding XM (2001) Establishment of gene replacement/disruption system in Amycolatopsis mediterranei U32. Ph. D. Dissertation. Institute of Plant Physiology and Ecology, Shanghai Institutes for Biological Sciences, Chinese Academy of Sciences

15. Hillemann D, Dammann T, Hillemann A, Wohlleben W (1993) Genetic and biochemical characterization of the two glutamine synthetases GSI and GSIl of the phosphinothricyl-alanyl-alanine producer, streptomyces viridochromogenes Tu494. J Gen Microbiol 139:1773-1783

16. Yu H, Peng WT, Liu Y, Wu T, Yao YF, Cui MX et al (2006) Identification and characterization of glnA promoter and its corresponding trans-regulatory 
protein GInR in the rifamycin SV producing actinomycete, Amycolatopsis mediterranei U32. Acta Biochim Biophys Sin (Shanghai) 38:831-843

17. Wang Z, Gerstein M, Snyder M (2009) RNA-seq: a revolutionary tool for transcriptomics. Nat Rev Genet 10:57-63

18. Agarwal A, Koppstein D, Rozowsky J, Sboner A, Habegger L, Hillier LW et al (2010) Comparison and calibration of transcriptome data from RNAseq and tiling arrays. BMC Genomics 11:383

19. Oshlack A, Robinson MD, Young MD (2010) From RNA-seq reads to differential expression results. Genome Biol 11:220

20. McClure R, Balasubramanian D, Sun Y, Bobrovskyy M, Sumby P, Genco CA et al (2013) Computational analysis of bacterial RNA-seq data. Nucleic Acids Res 41:e140

21. McClure R, Tjaden B, Genco C (2014) Identification of sRNAs expressed by the human pathogen Neisseria gonorrhoeae under disparate growth conditions. Front Microbiol 5:456

22. Delihas N (1995) Regulation of gene expression by trans-encoded antisense RNAs. Mol Microbiol 15:411-414

23. Storz G, Opdyke JA, Zhang A (2004) Controlling mRNA stability and translation with small, noncoding RNAs. Curr Opin Microbiol 7:140-144

24. Peng WT, Wang J, Wu T, Huang JQ, Chiao JS, Zhao GP (2006) Bacterial type I glutamine synthetase of the rifamycin SV producing actinomycete, Amycolatopsis mediterranei U32, encoded by $g \ln A$, is the only enzyme responsible for glutamine synthesis under physiological conditions. Acta Biochem Biophys Sin 38:821-830

25. Zhang W, Yang L, Jiang W, Zhao G, Yang Y, Chiao J (1999) Molecular analysis and heterologous expression of the gene encoding methylmalonylcoenzyme A mutase from rifamycin SV-producing strain Amycolatopsis mediterranei U32. Appl Biochem Biotechnol 82:209-225

26. Meier TW, Thoma NH, Leadlay PF (1996) Tritium isotope effects in adenosylcobalamin-dependent methylmalonyl-CoA mutase. Biochemistry 35:11791-11796

27. Reeves AR, Brikun IA, Cernota WH, Leach BI, Gonzalez MC, Weber JM (2006) Effects of methylmalonyl-CoA mutase gene knockouts on erythromycin production in carbohydrate-based and oil-based fermentations of Saccharopolyspora erythraea. J Ind Microbiol Biotechnol 33:600-609

28. Reeves AR, Brikun IA, Cernota WH, Leach BI, Gonzalez MC, Weber JM (2007) Engineering of the methylmalonyl-CoA metabolite node of Saccharopolyspora erythraea for increased erythromycin production. Metab Eng 9:293-303

29. Peano C, Damiano F, Forcato M, Pietrelli A, Palumbo C, Corti G et al (2014) Comparative genomics revealed key molecular targets to rapidly convert a reference rifamycin-producing bacterial strain into an overproducer by genetic engineering. Metab Eng 26C:1-16

30. Guo J, Frost JW (2002) Kanosamine biosynthesis: a likely source of the aminoshikimate pathway's nitrogen atom. J Am Chem Soc 124:10642-10643
31. Arakawa K, Muller R, Mahmud T, Yu TW, Floss HG (2002) Characterization of the early stage aminoshikimate pathway in the formation of 3-amino5-hydroxybenzoic acid: the RifN protein specifically converts kanosamine into kanosamine 6-phosphate. J Am Chem Soc 124:10644-10645

32. Absalon AE, Fernandez FJ, Olivares PX, Barrios-Gonzalez J, Campos C, Mejia A (2007) RifP; a membrane protein involved in rifamycin export in Amycolatopsis mediterranei. Biotechnol Lett 29:951-958

33. Lancini G, Cavalleri B (1997) In biotechnology of antibiotics. Marcel Dekker, New York, p 521

34. Bentley SD, Chater KF, Cerdeno-Tarraga AM, Challis GL, Thomson NR, James KD et al (2002) Complete genome sequence of the model actinomycete Streptomyces coelicolor A3(2). Nature 417:141-147

35. Serrano A, Losada M (1988) Action spectra for nitrate and nitrite assimilation in blue-green algae. Plant Physiol 86:1116-1119

36. Atkinson DE, McNall EG (1956) Nitrate reduction. I. Growth of Escherichia coli with nitrate as sole source of nitrogen. J Bacteriol 72:226-229

37. Rachmilevitch S, Cousins AB, Bloom AJ (2004) Nitrate assimilation in plant shoots depends on photorespiration. Proc Natl Acad Sci USA 101:11506-11510

38. Jenkins VA, Barton GR, Robertson BD, Williams KJ (2013) Genome wide analysis of the complete GInR nitrogen-response regulon in Mycobacterium smegmatis. BMC Genomics 14:301

39. Tiffert Y, Supra P, Wurm R, Wohlleben W, Wagner R, Reuther J (2008) The Streptomyces coelicolor GInR regulon: identification of new $\mathrm{Gln} R$ targets and evidence for a central role of GInR in nitrogen metabolism in actinomycetes. Mol Microbiol 67:861-880

40. Mejia A, Barrios-Gonzalez J, Viniegra-Gonzalez G (1998) Overproduction of rifamycin B by Amycolatopsis mediterranei and its relationship with the toxic effect of barbital on growth. J Antibiot (Tokyo) 51:58-63

41. Ferree MA, Shannon RD (2001) Evaluation of a second derivative UV/ visible spectroscopy technique for nitrate and total nitrogen analysis of wastewater samples. Water Res 35:327-332

42. Yao Y, Zhang W, Jiao $R$, Zhao G, Jiang W (2002) Efficient isolation of total RNA from antibiotic-producing bacterium Amycolatopsis mediterranei. J Microbiol Methods 51:191-195

43. Langmead B, Salzberg SL (2012) Fast gapped-read alignment with Bowtie 2. Nat Methods 9:357-359

44. Quinlan AR, Hall IM (2010) BEDTools: a flexible suite of utilities for comparing genomic features. Bioinformatics 26:841-842

45. Wang LK, Feng Z, Wang X, Wang XW, Zhang XG (2010) DEGseq: an R package for identifying differentially expressed genes from RNA-seq data. Bioinformatics 26:136-138

\section{Submit your next manuscript to BioMed Central and take full advantage of:}

- Convenient online submission

- Thorough peer review

- No space constraints or color figure charges

- Immediate publication on acceptance

- Inclusion in PubMed, CAS, Scopus and Google Scholar

- Research which is freely available for redistribution

Submit your manuscript at 